'Department of Management, Politics and Philosophy, Copenhagen Business School, Frederiksberg, Denmark

${ }^{2}$ The Research Unit for General Practice and Section of General Practice, Department of Public Health, University of Copenhagen, Copenhagen, Denmark

${ }^{3}$ Department of Social and Decision Sciences, Carnegie Mellon University, Pittsburgh, Pennsylvania, USA ${ }^{4}$ Department of Engineering and Public Policy, Carnegie Mellon University, Pittsburgh,

Pennsylvania, USA

\section{Correspondence to} Professor Laurel Austin, Department of Management, Politics and Philosophy, Porcelaenshaven 18A Copenhagen Business School, 2000 Frederiksberg, Denmark; lau.lpf@cbs.dk

Accepted 8 October 2011 Published Online First 16 November 2011

\title{
Injury prevention and risk communication: a mental models approach
}

\author{
Laurel C Austin, ${ }^{1,2}$ Baruch Fischhoff ${ }^{3,4}$
}

\begin{abstract}
Individuals' decisions and behaviour can play a critical role in determining both the probability and severity of injury. Behavioural decision research studies peoples' decision-making processes in terms comparable to scientific models of optimal choices, providing a basis for focusing interventions on the most critical opportunities to reduce risks. That research often seeks to identify the 'mental models' that underlie individuals' interpretations of their circumstances and the outcomes of possible actions. In the context of injury prevention, a mental models approach would ask why people fail to see risks, do not make use of available protective interventions or misjudge the effectiveness of protective measures. If these misunderstandings can be reduced through context-appropriate risk communications, then their improved mental models may help people to engage more effectively in behaviours that they judge to be in their own best interest. If that proves impossible, then people may need specific instructions, not trusting to intuition or even paternalistic protection against situations that they cannot sufficiently control. The method entails working with domain specialists to elicit and create an expert model of the risk situation, interviewing lay people to elicit their comparable mental models, and developing and evaluating communication interventions designed to close the gaps between lay people and experts. This paper reviews the theory and method behind this research stream and uses examples to discuss how the approach can be used to develop scientifically validated context-sensitive injury risk communications.
\end{abstract}

\section{ROLE OF MENTAL MODELS IN INJURY RISK AND PREVENTION}

With more focus by health authorities and researchers, the toll of injuries is becoming better understood. In the EU, injury is the fourth leading cause of death overall, with many times as many non-fatal injuries. ${ }^{1}$ World Health Organization data estimate that about $8 \%$ of deaths worldwide in 2004 were due to fatal injury (excluding war), with $75 \%$ unintentional. ${ }^{2}$ In the USA, although unintentional injury accounts for $<10 \%$ of deaths, it accounts for about $30 \%$ of years of life lost before the age of 75 (Arias, ${ }^{3}$ unpublished).

Injuries generally occur in dynamic situations where people respond to real-time information and conditions. Passive interventions that do not require behaviour change are often more effective than active interventions that do. ${ }^{4}$ However, passive interventions are not available for all injury situations, and sometimes legal requirements are rejected as overly paternalistic. When passive interventions are available and accepted, individuals' decisions and behaviours still affect their use and thus injury probability and severity. ${ }^{5}$ Designing communications to improve decision making and safe behaviour is therefore an essential component of injury prevention.

We propose using the 'mental models' method of risk communication-successfully used in health and environmental risk domains - in the domain of injury prevention. As in these domains, injury risk exists within an interactive system involving people, groups, institutions, policies and physical settings. $^{8-10} \mathrm{i}$ In the injury prevention literature and in public health literature more generally, this system is conceptualised as consisting of a host (person who faces injury risk), the agent or vehicle (which transmits the risk) and the physical and social environments impacting the host and agent. ${ }^{11}$ A successful risk communication research strategy in these other areas has been to contrast formal 'expert' models of those processes with intuitive mental models, and to develop communications to improve those mental models. ${ }^{12-14}$ The methodology has been applied in many domains, including radon in the home,${ }^{15}$ pathogens in drinking water, ${ }^{16}$ sexually transmitted diseases (STDs), ${ }^{17}$ breast cancer, ${ }^{18}$ breast implants, ${ }^{19}$ vaccines $^{20}$ and natural resource management, ${ }^{21}$ among others.

An individual's mental model of a dynamic system is a simplified cognitive representation of a complex system that exists in the world. ${ }^{22}$ ii It consists of relevant knowledge and beliefs, including perceptions of interconnectedness. It may be called upon over time for making inferences, changing in detail with time, experience and context. Once assembled, mental models affect how subsequent information is processed. ${ }^{23}$ Information consistent with existing beliefs is more easily acquired and integrated, strengthening those beliefs, whether accurate or not. Where people have had effective instruction, their beliefs might largely approximate those of the experts, albeit with less depth. Where they lack instruction, people draw upon whatever comes to mind-including personal experience, formal education and media communications-to devise and evaluate possible decision options.

'We use the term 'risk' as in risk management research where a risk has two dimensions: probability and magnitude. For discussions of the definitions of risk see Aven and Renn ${ }^{6}$ and Fischhoff and Kadvany. ${ }^{7}$

"Doyle and Ford ${ }^{22}$ define a mental model of a dynamic system as 'a relatively enduring and accessible, but limited, internal conceptual representation of an external system whose structure maintains the perceived structure of that system'. 
In the context of injury prevention, mental models can lead people to make poor choices because:

- The actions seem so natural that they are taken without thinking.

- Gaps or misconceptions lead to underestimating risks or overestimating effectiveness of protective measures.

- People cannot understand instructions well enough to heed or follow them.

- People fail to recognise changes in their circumstances that affect injury risk.

\section{MENTAL MODELS APPROACH TO RISK COMMUNICATION}

In such cases, people need help in refining their mental models. Yet it is recognised in the injury prevention literature that limited communication between researchers, practitioners and lay people, as well as insufficient sensitivity to contextual factors and actual knowledge needs when using a public health approach to injury prevention, contributes to difficulties in translating research findings into practice. This is known as a research-to-practice gap. ${ }^{102425}$ The mental models approach to risk communication addresses barriers to getting needed expertise to lay decision makers. The theory starts with the foundational assumption of behavioural decision theory that an individual's beliefs influence his or her decisions, which influence behaviours. The approach integrates two research traditions: (1) elicitation of expert beliefs (to perform the formal analysis); and (2) mental models research (to describe and address lay beliefs), and requires five steps:

- Elicit domain expert beliefs and integrate them in a formal model of the risk situation.

- Elicit lay beliefs about the same domain.

- Compare expert and lay beliefs to identify consequential gaps and misperceptions.

- Use structured surveys to estimate the population prevalence of beliefs.

- Develop and empirically evaluate contextually relevant communications.

Here we highlight the essentials of the method, although it is not possible to provide a detailed methods section for each step (more details of the methodology are given by Morgan et $a l^{13}$ ). We then review some previous research that might best convey relevance to injury prevention researchers.

\section{Step 1: Eliciting and modelling expert beliefs}

Eliciting and modelling expert beliefs starts with a review of relevant literature to identify the key factors (or variables) in the system and relationships among them. These factors are not only risk factors as found in epidemiological risk models, but also institutional factors, contextual factors, social factors, communications, etc. The factors are integrated in the form of an 'influence diagram', ${ }^{26} 27$ a form of directed network in which each node represents a factor and each connecting arrow represents a predictive (possibly causal) relationship between factors. In an influence diagram, oval nodes represent uncertain states or events and rectangular nodes represent decisions by the decision maker.

The initial model is usually at a fairly aggregated level called a high-level expert model. Each factor in such a model has its own predictors which can be captured in more detailed submodels. An expert model should have the conceptual precision needed to derive quantitative predictions, were its data needs satisfied. Often, though, the formalisation and integration meet the research needs. The goal of numerical prediction can even undermine understanding if it disproportionately favours readily quantified variables. $^{28}$

Figure 1 provides a basic template for an influence diagram of injury risk processes adapted from Morgan et al. ${ }^{13}$ This shows that pre-event prevention activities may influence environmental, agent and host factors which, in turn, affect the probability and severity of the injury event. The event and eventrelated prevention activities can affect environmental and host factors, as well as injury processes. These factors, together with post-event injury prevention activities, influence final outcomes.

Although they do not use the language of influence diagrams and expert models, Juarez et al developed a model of influences on teenage driving decisions and behaviours based on a literature review. ${ }^{8}$ It is reproduced in figure $2 \mathrm{~A}$ with appropriate modifications to illustrate an example of a preliminary expert model of influences on driving decisions and possible injury. In figure $2 \mathrm{~B}$ we create an illustrative example of a more detailed sub-model of individual factors and social influences on driving decisions.

Once a preliminary model has been created, it undergoes iterative evaluation with domain experts, sometimes meeting more than once with each expert. This may involve one-on-one semi-structured interviews or discussion with a panel of experts. Discussions often include a visual representation of the preliminary model. Experts help develop more detailed sub-models.

Experts should represent the range of views on relevant topics. For injury prevention they would include epidemiologists who understand risk factors, experts in the technologies and environments creating or mitigating risks, policy analysts
Figure 1 A basic template for an influence diagram of injury risk processes. Adapted from Morgan et $\mathrm{al}^{13}$ and reprinted with permission from Cambridge University Press.

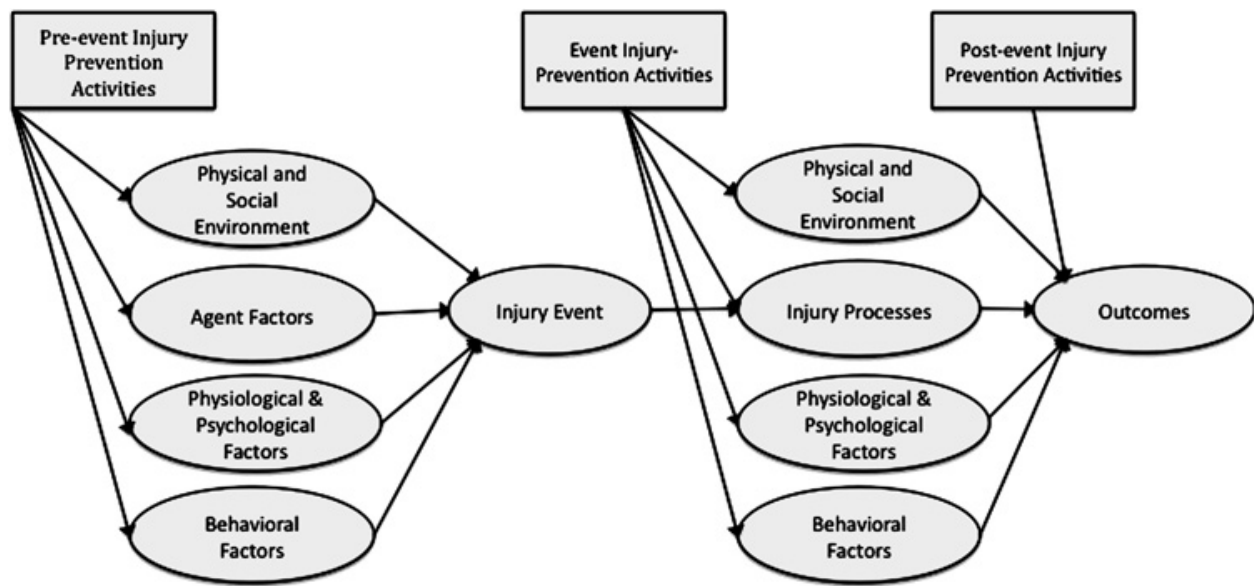


Figure 2 (A) An example of an influence diagram depicting a preliminary expert model of driving behaviour and vehicle crash, adapted from Juarez et $a l^{8}$ by permission from BMJ Publishing Group Ltd. (B) An example of a detailed sub-model illustrating influences of individual driver characteristics and social factors on some driver decisions, based on Juarez et al.'s literature review.
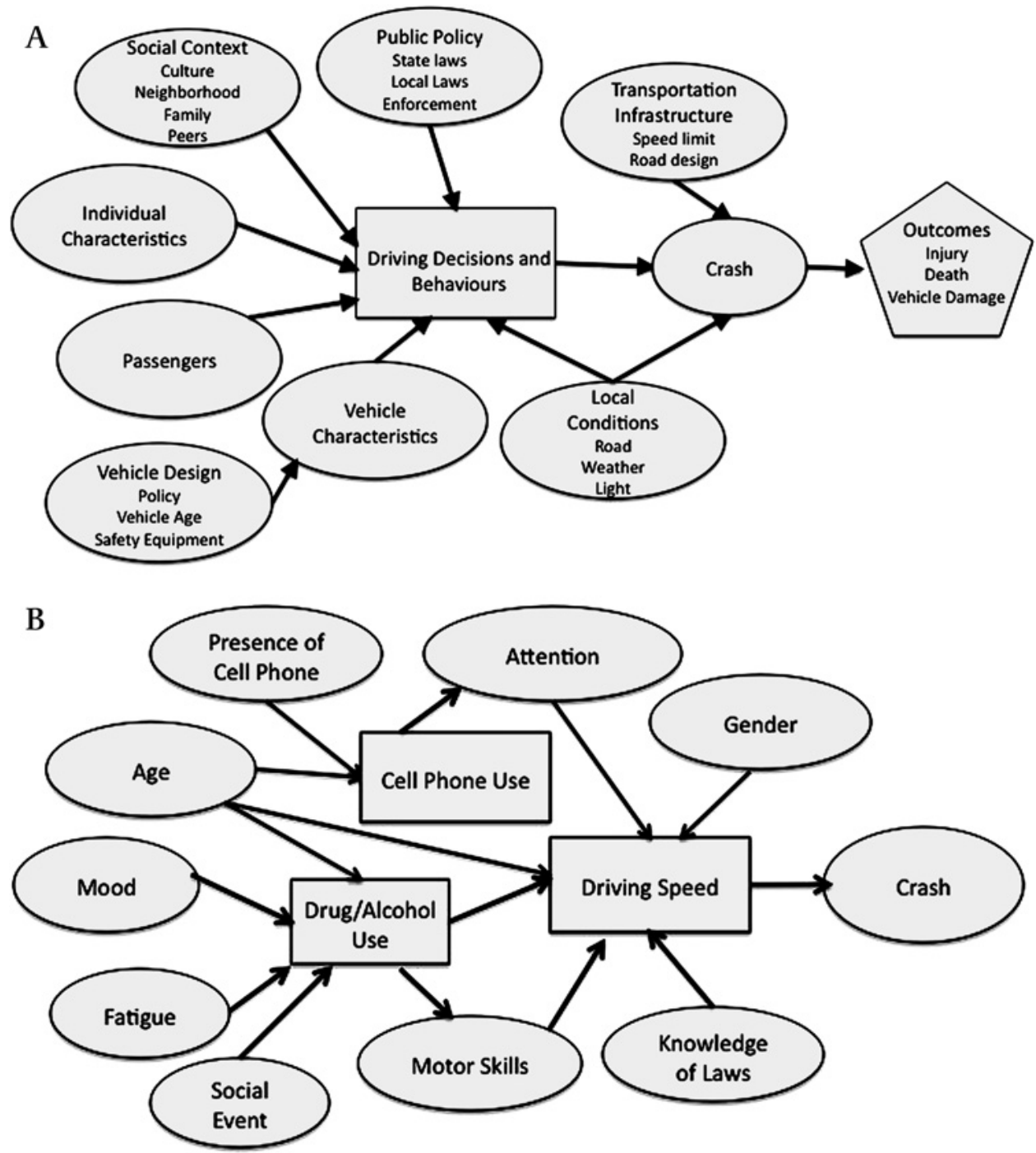

knowledgeable in institutional determinants and social scientists familiar with research into individual and social aspects of injury-related behaviour. The expert model is a composite of expert knowledge and beliefs, and is not one assumed to exist in the head of any one expert. It may include contradictory beliefs and uncertainties, if that is the state of the science, and miss variables that researchers have not studied.

\section{Step 2: Eliciting lay beliefs}

This involves in-depth individual interviews following a protocol developed around the expert model. The goal is to elicit beliefs in terms that are natural to respondents. Individual interviews are preferred to focus group interviews because the behaviours in question are generally at the individual level and because, in focus groups, a few people can influence others' thoughts or propensity to speak, leading to incomplete or inaccurate elicitation.

Interviews are recorded and transcribed in order to help researchers to hear exactly what each respondent says, as well as to allow independent coding of the interviews. Interviews start with open-ended questions ("What do you know about escalators?") in order to avoid suggesting specific ideas or wording. Respondents are asked to elaborate on whatever they say. Once respondents have exhausted these self-generated thoughts, they are asked semi-structured questions about each concept in the expert model, again in general terms ("What might be special risks for children?"). The interview might conclude with structured questions, like those on surveys, with a request to explain the answers ("On a five-point scale, how useful are the emergency buttons on escalators? Why do you say that?"). Because such questions can be reactive (in the sense of affecting respondents' thinking), positioning them at the end of the interview keeps them from affecting responses to the initial open-ended questions meant to capture the most natural and spontaneous thoughts.

In a homogeneous population, a sample of 20 individuals affords roughly a $50 \%$ chance of observing each belief held by at least $5 \%$ of a population. From this perspective, a sample of 20-30 should reveal all beliefs that are at least somewhat common. Ethnographic researchers have found that no new ideas are mentioned after as few as 12 interviews. ${ }^{29}$ For these reasons, and because of the high cost of interview research, mental models interview samples typically include $20-30$ respondents.

\section{Step 3: Analysing interview transcripts to identify gaps and misperceptions}

The third step in the method involves analysing interview transcripts to identify gaps and misperceptions in both lay understanding and the expert model. Although, by definition, experts know more than lay people, eliciting lay beliefs can reveal variables and relationships missing from expert 
conceptualisations. Sometimes those additional concerns reflect misunderstandings that communications can address, sometimes they reflect topics that might be relevant but have yet to be studied scientifically, and sometimes they reflect contexts or outcomes that experts have tended to neglect but which could be studied (eg, "If people are concerned about seeming alarmist, we can help to create social norms that will legitimate asking for help").

Interview analysis involves coding statements in responses into the variables and relationships in the expert model. Responses that do not fit are used to create an auxiliary list of concepts that can be evaluated in terms of whether they are missing from the expert model or irrelevant to it. Having two individuals independently code transcripts allows assessment of the reliability of the process. ${ }^{30}$ The conceptual clarity of the expert model typically allows high coding reliability.

Using both a formal expert model and open-ended interviews represents a compromise between the complementary methods of highly structured surveys, which can miss important lay beliefs and intuitive modes of expression but sample large groups and support statistical analyses, ${ }^{31-33}$ and fully qualitative research which can capture lay beliefs in ways that capture their fuller social context but make it hard to evaluate and improve their accuracy. ${ }^{34}$

\section{Step 4: Structured surveys}

The fourth step involves structured surveys with large samples to assess the prevalence of beliefs as suggested in the interviews. This method avoids limiting investigation only to concepts thought relevant by a handful of researchers or domain experts; in particular, such experts may not be sufficiently aware of contextual factors or personal motivations that influence realtime behaviours. Large sample size allows for quantitative analyses to determine prevalence of beliefs, context differences (workers in a mine versus a factory), population differences (adolescents versus adults), etc. More details on survey research methods are discussed by, for example, Converse and Presser ${ }^{35}$ and Krosnick. ${ }^{36}$

\section{Step 5: Developing context-appropriate risk communications}

The fifth step involves developing context-appropriate risk communications to correct important gaps and misperceptions while reinforcing appropriate beliefs. Interview transcripts suggest context and wording that will resonate with the intended audience(s). Empirically testing communications determines whether they are understandable and sufficiently correct gaps and misperceptions. One of the first mental models studies considered radon risk communication. ${ }^{15} 37$ The researchers evaluated two communications they developed and a widely disseminated Environmental Protection Agency brochure. ${ }^{38}$ Evaluation methods included think-aloud protocol analysis, ${ }^{39}$ problem solving and a series of true/false questions to assess agreement with the expert model after reading a communication. The mental model's communications were less confusing to readers, and those who read them had beliefs more consistent with the expert model than those who read the standard brochure. For methods for developing and testing communications, see Atman et al, ${ }^{37}$ Bostrom et $a l^{38}$ and chapter 6 of Morgan et al. ${ }^{13}$

\section{HOW THE APPROACH HAS BEEN USED}

We now consider two studies chosen to convey variation in the use of the method and because they might especially resonate with injury prevention researchers. The study by Cox et al may be situated within the injury control domain in that the focus is occupational health and safety communications. ${ }^{40}$ Their expert models were focused on particular agents (chemicals). Although possible harms were acute and chronic health effects, the methodology would have been the same had the effects included explosions and burns or other injuries. The problem formulation of the study by Downs et $a l^{17}$ is structurally similar to many injury prevention problems in that the focus was a specific harm in a specific context (STDs and adolescents), where the probability of harm is greatly affected by individual behaviours and use of prevention. Researchers interested in structuring research around injury-causing events (car crashes, falls from trees) might look for specific methods from previous research that starts with an expert model of an event such as vaccination ${ }^{20}$ or floods and landslides. $^{41}$

Cox et al developed a generic method for designing contextappropriate occupational safety communications. ${ }^{40}$ They considered the use of perchloroethylene in dry cleaning and rosin-based solder flux in the electrical/electronics sector in order to examine organisational and social context effects on communication and understanding of safety information. Injury prevention researchers increasingly note a need to understand how context impacts on injury. ${ }^{10} 25$ After developing a preliminary expert model from relevant literature, experts modelled their own beliefs in influence diagrams and then commented on the preliminary model. All this was synthesised into an expert model for each agent.

Interviews were conducted with 10 perchloroethylene users and 11 solder flux users. Cox et al did not code the transcripts as described above but used a grounded theory approach to qualitative data analysis to develop a hierarchical list of concepts mentioned by respondents, arguing that this approach better elicits contextual influences. Those concepts were compared with concepts in the expert model and analysed for gaps, misperceptions and correct user knowledge in lay responses.

The researchers then designed work-context dependent communications for the two sectors. ${ }^{42}$ They used a multipronged approach to evaluate those communications, standard material safety data sheets (MSDS) which chemical manufacturers must produce and distribute in the EU with every chemical used, and safety leaflets produced by the UK Health and Safety Executive. Users viewed MSDS as useful to emergency responders while Health and Safety Executive leaflets were viewed as more useful to users themselves. The researchers concluded that standard MSDS did not adequately inform workers about how to protect themselves, in part because the information is provided in a generic way. Users responded better to the mental models communications where information was specific to their work context. They found users related best of all to the mental models communications, that the method was good at filling important gaps, but that it was difficult to correct existing misperceptions.

Downs and colleagues used an expert model of the risk of STD to create an interactive DVD that led to reduced STD risks among sexually active adolescent girls. ${ }^{17}{ }^{43}$ In this case the host would be a teenage girl, an event would be sexual intercourse, the agent would be a bacteria or virus and the effects would be health effects, specifically STDs. Active preventive measures could be taken prior to the event (techniques to avoid sexual activitity), during the event (eg, use of a condom) and after the event (medical intervention to prevent progress of an existing STD)

These investigators have now begun a large-scale trial involving 3000 young women using the DVD under normal 
clinic conditions. The expert model is built around the factors affecting teenagers' choices regarding behaviours that affect disease risk, developed with a panel of experts in public health, adolescent medicine, nursing and psychology. A key finding in mental models interviews with 48 adolescent girls was that they often failed to see the decisions leading to sexual acts or to have a feeling of control over those decisions. They also knew little about reproductive health or STDs other than HIV/AIDS.

Downs et $a l^{17}$ designed three interventions with the same context, focusing on gaps and misperceptions in adolescent girls' mental models: an interactive video, a book with the dialogue and some images from the video, and brochures covering the same topics secured from existing commercial sources. The video and book portrayed adolescent girls with boyfriends in situations that might lead to a sexual encounter. Viewers chose which sections to watch and encountered several choice points where the video froze for $30 \mathrm{~s}$ and encouraged viewers to think about possible choices, with an actress then modelling one way to control the situation. Another section showed an older sister teaching a younger sister how to secure and use condoms. The book adopted these strategies to its format. It is easy to imagine how such interactive communications could be useful in the domain of injury prevention, perhaps directed at different types of people (girls vs boys) or different contexts for the same kind of injury.

The researchers conducted a longitudinal controlled study to evaluate the impact of the intervention. In that study of 300 girls, those who viewed the interactive video reported fewer condom failures 3-6 months later than those in the control group. After 6 months they were significantly less likely to report an STD diagnosis than girls in either of the two control groups.

\section{DISCUSSION}

Research using the mental models approach initially focused on environmental and natural hazards. Individuals cannot control whether such hazards occur, but can prevent or limit harm if they do. For example, one cannot eliminate radon sources from under one's home, but can respond if it is known or suspected to be there. Mental models research has gradually expanded to health risks where individual choices may play important roles in disease acquisition, diagnosis and treatment.

Compared with environmental and many health risks, injury situations often offer more opportunities for individual choices to alter the probability or severity of adverse events through protective actions, emergency responses or preventive vigilance. For example, defensive driving can reduce both the probability and severity of a collision, while pre-emptively buckling seat belts can reduce the probability and severity of being injured if a collision occurs. These individual behaviours often take place in complex dynamic systems involving people, processes and technologies. Extending the mental models approach to these domains would both advance the method by addressing these novel problems, and provide a new perspective on them, applying scientific knowledge (eg, from epidemiology) to improving lay understanding. The expert models themselves can provide the foundation for systematic training designed to afford people with mental models that allow them to analyse and respond to emerging situations. For example, Bruine de Bruin et al found that an expert model outperformed narrative instruction, using the same material, when explaining the risks and benefits of xenotransplantation. ${ }^{44}$ Conversely, better descriptions of lay beliefs in risk-relevant terms might enhance scientific modelling. For example, Casman et al were able to prioritise passive and active risk management strategies through analyses that sought to inform lay decisions. ${ }^{16}$

We suggest that use of this method in injury prevention would be one way of addressing the concern that the public health approach does not sufficiently consider the role of context and knowledge needs in injury prevention. The method readily lends itself to the development of different communications by assessing the prevalence of beliefs in different audiences and targeting context-appropriate communications to each. Audiences might be defined by relevant demographic factors, by the context in which they face injury risk or by some measure of expertise. $^{21}$

A limitation of mental models research in the public health and environmental health domains is that it is difficult to assess the impact of the interventions on outcomes realised in the distant future. For example, the effects of radon are not known for many years and conducting a controlled intervention study is not feasible. The work by Downs et al on STDs is an exception and showed that adolescents communicated to with the mental models approach experienced fewer harms than adolescents in the control groups. Applying the method in the domain of injury prevention might offer more opportunity for controlled study of intervention in risk situations over a reasonably short period of time, enhancing opportunities to assess the efficacy of the method.

The mental models method can be used for both persuasive and non-persuasive approaches to injury prevention. ${ }^{45}$ On the one hand it can identify facts that might be used to convince people to take injury prevention measures while, on the other, it can identify the facts that will enable people to evaluate which measures they want to take. Whatever the communication strategy, creating an expert model helps to ensure that prevention practitioners are staying true to the science, including its uncertainties. Conducting mental models interviews helps to ensure that the facts are presented in the most cogent way possible.

Like any other descriptive research, mental models studies can only suggest ways to improve individuals' decisions regarding injury-related behaviours. Acting on (and testing) those suggestions requires concrete interventions, carefully developed with input from potential users, in order to produce the best possible realisation of that theoretical understanding. Like all interventions, these must be evaluated empirically in order to assess success. Injury prevention risk communications are not only relevant when communicating about active prevention through behaviour change but also for communications related to the use of institutional or passive preventive measures-for example, persuading use of a legally required measure or dissuading circumvention of new unfamiliar safety features in equipment or vehicles. To the extent that contextually and scientifically appropriate communications that reinforce correct beliefs and address gaps and misperceptions in knowledge can help people make better decisions and manage the risks they face, we believe the approach described here would be beneficial.

Acknowledgments The authors would like to thank our anonymous reviewers for their very thoughtful comments on an earlier version of this manuscript.

Competing interests None.

Provenance and peer review Not commissioned; externally peer reviewed.

\section{REFERENCES}

1. Injuries in the European Union. Statistics Summary 2005-2007. Vienna: EuroSafe, 2009. http://www.eurosafe.eu.com/csi/eurosafe2006.nsf/wwwFreeText/ 2DD3B414D49544AEC1257686004E6EBC?OpenDocument\&11122009151434 
2. Health Statistics and Health Information Systems. Disease and Injury Country Estimates, Burden of Disease. World Health Organization. 2009. http://www.who.int/ healthinfo/global burden disease/estimates country/en/index.html

3. Arias I. Making a difference: CDC's investment in injury and violence prevention and lessons learned for the global context. Safety 2010 World Conference. London.

4. Christoffel T, Gallagher SS. Injury Prevention and Public Health: Practical Knowledge, Skills, and Strategies. 2nd edn. Sudbury, Mass: Jones and Bartlett, 2006.

5. Gielen AC, Sleet D. Application of behavior-change theories and methods to injury prevention. Epidemiol Rev 2003:25:65-76.

6. Aven T, Renn 0. On risk defined as an event where the outcome is uncertain. J Risk Res 2009;12:1-11.

7. Fischhoff B, Kadvany J. Risk: A Very Short Introduction. New York: Oxford University Press, 2011

8. Juarez $\mathbf{P}$, Schlundt DG, Goldzweig I, et al. A conceptual framework for reducing risky teen driving behaviors among minority youth. Inj Prev 2006;12(Suppl 1):i49-55.

9. McClure RJ. Injury risk and prevention in context. Inj Prev 2010;16:361-2.

10. McClure RJ, Davis E, Yorkston E, et al. Special issues in injury prevention research: developing the science of program implementation. Injury 2010;41(Suppl 1):S16-19.

11. Runyan CW. Introduction: back to the future-revisiting Haddon's conceptualization of injury epidemiology and prevention. Epidemiol Rev 2003:25:60-4.

12. Fischhoff B. Decision research strategies. Health Psychol 2005;24(4 Suppl):S9-16

13. Morgan MG, Fischhoff B, Bostrom A, et al. Risk Communication: A Mental Models Approach. New York: Cambridge University Press, 2002.

14. Rouse WB, Morris NM. On looking into the black box: prospects and limits in the search for mental models. Psychol Bull 1986;100:349-63.

15. Bostrom A, Fischhoff B, Morgan MG. Characterizing mental models of hazardous processes: a methodology and an application to radon. J Soc Issues 1992;48:85-100.

16. Casman EA, Fischhoff B, Palmgren $\mathrm{C}$, et al. An integrated risk model of a drinking water-borne cryptosporidiosis outbreak. Risk Anal 2000;20:495-512.

17. Downs JS, Murray PJ, Bruine de Bruin W, et al. Interactive video behavioral intervention to reduce adolescent females' STD risk: a randomized controlled trial. Soc Sci Med 2004;59:1561-72.

18. Silverman E, Woloshin S, Schwartz LM, et al. Women's views on breast cancer risk and screening mammography: a qualitative interview study. Med Decis Making 2001;21:231-40.

19. Byram S, Fischhoff B, Embrey M, et al. Mental models of women with breast implants: local complications. Behav Med 2001;27:4-14.

20. Downs JS, Bruine de Bruin WB, Fischhoff B. Parents' vaccination comprehension and decisions. Vaccine 2008;26:1595-607.

21. Zuksek M, Arvai J. Toward improved communication about wildland fire: mental models research to identify information needs for natural resource management. Risk Anal 2004;24:1503-14.

22. Doyle JK, Ford DN. Mental models concepts for system dynamics research. Syst Dynam Rev 1998;14:3-29.

23. Fischhoff B. Cognitive processes in stated preference methods. In: Maler KG, Vincent JR, eds. Handbook of Environmental Economics: Valuing Environmental Changes. Amsterdam: Elsevier, 2005:937-68.
24. Bugeja L, McClure RJ, Ozanne-Smith J et al. The public policy approach to injury prevention. Inj Prev 2011;17:63-5.

25. Mallonee S, Fowler C, Istre GR. Bridging the gap between research and practice: a continuing challenge. Inj Prev 2006:12:357-9.

26. Burns WJ, Clemen RT. Covariance structure models and influence diagrams. Manag Sci 1993;39:816-34.

27. Howard RA. Knowledge maps. Manag Sci 1989;35:903-22

28. Fischhoff B, Bruine de Bruin W, Güvenç Ü, et al. Analyzing disaster risks and plans: an avian flu example. J Risk Uncertain 2006;33:131-49.

29. Guest G, Bunce A, Johnson L. How many interviews are enough? An experiment with data saturation and variability. Field Meth 2006;18:59-82.

30. Lombard M, Snyder-Duch J, Bracken CC. Content analysis in mass communication: assessment and reporting of intercoder reliability. Hum Comm Res 2002;28:587-604.

31. Bruce BS, Snowdon AW, Cunningham C, et al. Predicting parents' use of booster seats. Inj Prev 2011;17:313-18.

32. Gielen AC, Eriksen MP, Daltroy LH, et al. Factors associated with the use of child restraint devices. Health Educ Q 1984;11:195-206.

33. Peterson L, Farmer J, Kashani JH. Parental injury prevention endeavors: a function of health beliefs? Health Psychol 1990:9:177-91.

34. Reventlow SD, Hvas L, Malterud K. Making the invisible body visible: bone scans, osteoporosis and women's bodily experiences. Soc Sci Med 2006:62:2720-31.

35. Converse JM, Presser S. Survey Questions: Handcrafting the Standardized Questionnaire. Beverly Hills: Sage Publications, 1996.

36. Krosnick JA. Survey research. Annu Rev Psychol 1999:50:537-67.

37. Atman CJ, Bostrom A, Fischhoff B, et al. Designing risk communications: completing and correcting mental models of hazardous processes, Part 1. Risk Anal 1994:14:779-88

38. Bostrom A, Atman CJ, Fischhoff B, et al. Evaluating risk communications: completing and correcting mental models of hazardous processes, Part II. Risk Anal 1994;14:789-98

39. Ericsson KA, Simon HA. Protocol Analysis. Cambridge: MIT Press. 1992.

40. Cox P, Niewöhner J, Pidgeon N, et al. The use of mental models in chemical risk protection: developing a generic workplace methodology. Risk Anal 2003;23:311-24.

41. Wagner K. Mental models of flash floods and landslides. Risk Anal 2007;27:671-82

42. Niewöhner J, Cox P, Gerrard S, et al. Evaluating the efficacy of a mental models approach for improving occupational chemical risk protection. Risk Anal 2004;24:349-61.

43. Fischhoff B, Downs JS, Bruine de Bruin W. Adolescent vulnerability: a framework for behavioral interventions. Appl Prev Psychol 1998;7:77-94.

44. Bruine de Bruin W, Güvenç Ü, Fischhoff B, et al. Communicating about xenotransplantation: models and scenarios. Risk Anal 2009:29:1105-15.

45. Fischhoff B. Nonpersuasive communicaiton about matters of greatest urgency: climate change Environ Sci Technol 2007:41:7204-8.

Calendar

\section{Conferences and events of interest}

doi:10.1136/injuryprev-2012-040374

Edited by Barry Ivan Pless

Regional Safe Community Conferences 6th Asian Regional Conference on Safe Communities in 2012

11-13 May 2012, Tokyo, Japan.
The 3rd European Safe Community Conference The future role of the Fire and Rescue Service in the sustainability of Safe Communities in Europe

5 June 2012, Falköping, Sweden.

Canadian Multidisciplinary Road Safety Conference

10-13 June 2012, Banff, Alberta.

Australasian Conference on Road Safety

9-10 August 2012, Sydney, Australia. 11th World Conference on Injury Prevention and Safety Promotion

1-4 October 2012, Wellington, New Zealand.

\section{Association for the Advancement of Automotive Medicine}

14-17 October 2012, Seattle, Washington.

Canadian Injury Prevention and Safety Promotion Conference

6-9 November 2013, Montreal, Quebec. 